\title{
Investigation into the underlying molecular mechanisms of white adipose tissue through comparative transcriptome analysis of multiple tissues
}

\author{
SONG ZHANG ${ }^{1 *}$, LI WANG $^{1 *}$ and LINSEN ZAN ${ }^{1-3}$ \\ ${ }^{1}$ Key Laboratory of Animal Biotechnology, College of Animal Science and Technology; \\ ${ }^{2}$ Department of Agriculture Cattle Laboratory, National Beef Cattle Improvement Center; ${ }^{3}$ Molecular Breeding Laboratory, \\ Shaanxi Beef Cattle Engineering Research Center, Northwest A\&F University, Xianyang, Shaanxi 712100, P.R. China
}

Received April 8, 2018; Accepted November 14, 2018

DOI: $10.3892 / \mathrm{mmr} .2018 .9740$

\begin{abstract}
Adipose tissue has a primary role in lipid and glucose metabolism as a storage site for fatty acids, and also functions as an endocrine organ, producing large numbers of hormones and cytokines. Adipose dysfunction triggers a number of obesity-associated health problems. The aim of the present study was, therefore, to investigate the molecular mechanisms of white adipose tissue (WAT). The GSE9954 microarray data were downloaded from the Gene Expression Omnibus. Adipose-specific genes were identified through limma package analysis, based on samples of WAT and 17 other types of non-adipose tissue obtained from mice. Process and pathway enrichment analyses were performed for these genes. Finally, protein-protein interaction (PPI) and co-expression networks were constructed and analyzed. In total, 202 adipose-specific genes were identified, which
\end{abstract}

Correspondence to: Dr Linsen Zan, Key Laboratory of Animal Biotechnology, College of Animal Science and Technology, Northwest A\&F University, 22 Xinong Road, Yangling, Xianyang, Shaanxi 712100, P.R. China

E-mail: zanlinsen@163.com

*Contributed equally

Abbreviations: WAT, white adipose tissue; GEO, Gene Expression Omnibus; DEGs, differentially expressed genes; KEGG, Kyoto Encyclopedia of Genes and Genomes; GO, Gene Ontology; PPI, protein-protein interaction; MCODE, molecular complex detection; WGCNA, weighted gene co-expression network analysis; Acaca, acetyl-CoA carboxylase $\alpha$; Pparg, peroxisome proliferator-activated receptor $\gamma$; Lep, leptin; Fasn, fatty acid synthase; AMPK, adenine monophosphate-activated protein kinase; Retn, resistin; Cidec, cell death-inducing DFFA-like effector c; Slc27a1, solute carrier family 27 member 1; Aoc3, amine oxidase copper containing 3; Adrb3, adrenoceptor beta 3; VAP-1, enzyme vascular adhesion protein

Key words: white adipose tissue, Gene Expression Omnibus, microarray data, differentially expressed genes were involved in key biological processes (including fat cell differentiation and lipid metabolic process) and one key pathway [namely, the adenine monophosphate-activated protein kinase (AMPK) signaling pathway]. Construction of the PPI network and further molecular complex detection revealed the presence of 17 key genes, including acetyl-CoA carboxylase $\alpha$, peroxisome proliferator-activated receptor (PPAR) $\gamma$ and leptin, that were involved in the AMPK, PPAR and insulin signaling pathways. In addition, amine oxidase copper containing 3 and adrenoceptor beta 3 were communication hubs in the co-expression network of adipose-specific genes. In conclusion, the present study promotes our understanding of the underlying molecular mechanisms of WAT, and may offer an insight into the prevention and treatment of obesity-associated diseases caused by adipose dysfunction.

\section{Introduction}

The worldwide prevalence of obesity has almost tripled since 1975 (1). According to the most recent data reported by the World Health Organization (updated in October 2017), mortality associated with being overweight or obese as opposed to being underweight is greater in the vast majority of countries (1). Adipose tissue serves a primary role in lipid and glucose metabolism as a storage site for fatty acids, and produces a large number of hormones and cytokines as an endocrine organ (2). Adipose tissue dysfunction triggers a number of obesity-associated metabolic derangements, such as type 2 diabetes, insulin resistance and cardiovascular diseases $(2,3)$.

Cellular heterogeneity is a characteristic feature of adipose tissue, which contains several types of cells, including adipocytes, preadipocytes, fibroblasts, endothelial cells and multipotent stem cells (4). Adipocytes are the major component of adipose tissue and are considered to be the cornerstone of the steady-state control of systemic metabolism. Their main function is to control the energy balance by mobilizing triglycerides during times of energy starvation and storing triglycerides during periods of excessive energy. Preadipocytes undergo proliferation and differentiation, eventually forming mature adipocytes. This process determines 
the ability of adipose tissues to expand throughout the lifespan of humans (4), leading to central obesity (5). Adipocytes are replaced via the constant generation of new adipocytes, with renewal of nearly $50 \%$ of adipocytes in the human subcutaneous adipose tissue every 8 years (6). At the cellular level, an increase in the number and volume of adipocytes can fundamentally lead to hyperplastic and hypertrophic obesity, respectively (7). In severely obese individuals, the increase in the number of adipocytes is more prominent in comparison with the increase in the volume of adipocytes (8).

As the most abundant tissue in the body, white adipose tissue (WAT) serves a variety of physiological roles, mainly associated with metabolic and endocrine functions (9). The metabolic functions of WAT include the storage and mobilization of energy. Energy is stored in the body in the form of triglycerides through lipogenesis, while the stored triglycerides are hydrolyzed through lipolysis to meet the body's continuing energy needs (10). Specific enzymes acquired during adipocyte differentiation contribute to the two aforementioned processes, which are regulated by several pathways, including the insulin, adrenergic and atrial natriuretic hormone pathways (11). In addition, WAT is an endocrine organ that produces an abundance of peptides, proteins and lipids (9). Factors including hormones, cytokines, extracellular matrix components and fatty acid-derived products can act systemically in WAT and other tissues to influence metabolic homeostasis (9). Over the past several decades, a large number of studies have addressed the function of fat- and obesity-associated diseases. However, details regarding the molecular mechanisms of adipose tissue remain unclear. To thoroughly investigate the role of adipose tissue in energy homeostasis and obesity-associated diseases caused by adipose dysfunction, it is necessary to understand the key genes required for maintaining the normal physiological functions of WAT.

High-throughput molecular biology techniques, represented by gene chip technology, can provide large-scale gene expression data through the measurement of transcript abundance in diverse tissues or cells (12). The Gene Expression Omnibus (GEO) database stores originally submitted records obtained using common commercial arrays, such as Affymetrix, Agilent, Illumina or NimbleGen (12). Recently, there has been impressive progress in identifying and detailing nodes through various microarray data analyses of adipose tissue or adipocytes, and subsequent experimental verification $(13,14)$. However, few studies have attempted to identify the key genes in adipose tissue based on the comparative analysis of multiple tissues.

In view of the extremely close association between adipose tissue and obesity, the identification of fat-driven genes is particularly important. In obese individuals, intra-abdominal fat accumulation is more strongly associated with the development of related diseases as opposed to subcutaneous fat accumulation (15). Therefore, in the present study, the mRNA expression profiles of epididymal adipose tissue (a type of WAT) were compared with 17 types of non-adipose tissues in order to identify adipose-specific genes in WAT. These genes were then extensively analyzed using several bioinformatics methods, including process and pathway enrichment analyses, protein-protein interaction (PPI) network construction, molecular complex detection (MCODE)
Table I. Differentially expressed genes in adipose tissue compared with 17 non-adipose tissues.

\begin{tabular}{lcc}
\hline Group & $\begin{array}{c}\text { Upregulated } \\
\text { genes }\end{array}$ & $\begin{array}{c}\text { Downregulated } \\
\text { genes }\end{array}$ \\
\hline Adipose vs. adrenal gland & 2,149 & 2,266 \\
Adipose vs. bone marrow & 3,402 & 3,231 \\
Adipose vs. brain & 3,494 & 3,550 \\
Adipose vs. diaphragm & 3,073 & 3,568 \\
Adipose vs. eye & 3,137 & 3,031 \\
Adipose vs. heart & 3,143 & 2,890 \\
Adipose vs. kidney & 2,446 & 2,260 \\
Adipose vs. liver & 3,032 & 2,180 \\
Adipose vs. lung & 1,706 & 1,986 \\
Adipose vs. muscle & 2,891 & 2,565 \\
Adipose vs. pituitary gland & 2,783 & 3,136 \\
Adipose vs. salivary gland & 3,212 & 2,906 \\
Adipose vs. seminal vesicle & 2,843 & 2,671 \\
Adipose vs. small intestine & 3,152 & 2,551 \\
Adipose vs. spleen & 2,894 & 3,095 \\
Adipose vs. testis & 4,992 & 3,916 \\
Adipose vs. thymus & 3,098 & 3,060 \\
Adipose vs. all 17 tissues & 215 & 4 \\
\hline
\end{tabular}

analysis and co-expression network analysis. These analyses attempted to provide a more in-depth understanding of adipose performance at the molecular level, and to identify the key genes required for the function of WAT and metabolic disorders caused by adipose dysfunction.

\section{Materials and methods}

Microarray data. mRNA expression profiles from the GSE9954 data series were downloaded from the GEO database (16). The GSE9954 data series (Affymetrix Mouse Genome 430 2.0 Array; Affymetrix, Inc., Santa Clara, CA, USA) included the profiles of 58 wild-type tissue samples obtained from 10-12-week-old C57BL/6 male mice, including 3 epididymal adipose, 3 liver, 3 diaphragm, 3 salivary gland, 3 spleen, 4 muscle, 3 brain, 3 lung, 3 kidney, 3 adrenal gland, 4 bone marrow, 5 pituitary gland, 3 seminal vesicle, 3 thymus, 3 testis, 3 heart, 3 small intestine and 3 eye tissue samples.

Identification of differentially expressed genes (DEGs). The impute (Imputation for microarray data; www.bioconductor. org/packages/release/bioc/html/impute.html) (17) and limma (Linear Models for Microarray Data; www.bioconductor. org/packages/release/bioc/html/limma.html) (18) packages were used to identify DEGs between adipose and non-adipose tissues. The thresholds used in DEG screening were an adjusted P-value of $<0.05$ and a llog fold-change (FC)| of $>0.5$. All the packages used in the present study were deployed in the R programming language (version 3.3.3; https://www.r-project.org/).

Process and pathway enrichment analyses. For the functional annotation of the DEGs, process and pathway enrichment 


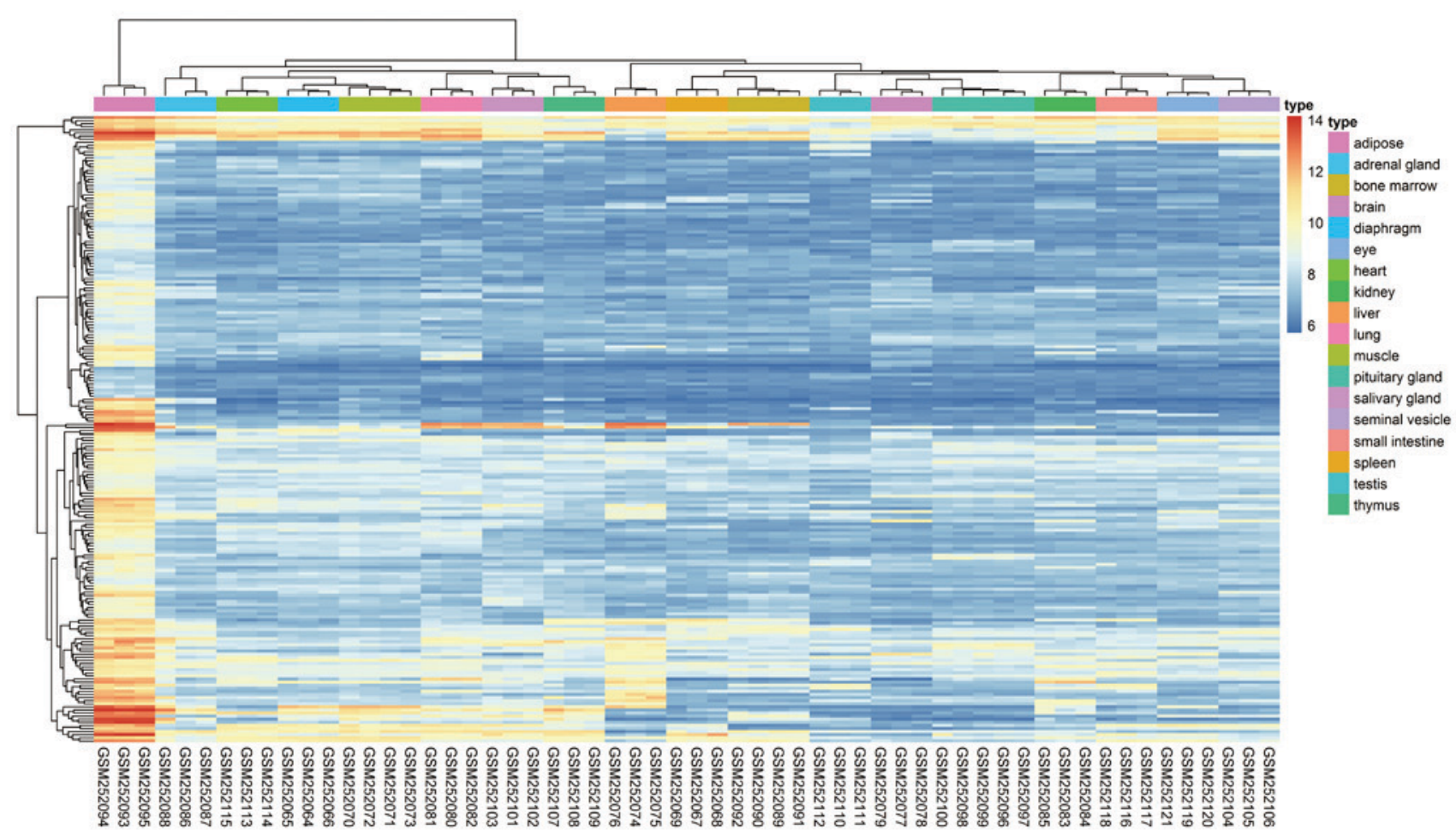

Figure 1. Hierarchical clustering analysis of adipose-specific genes in 58 samples from 18 tissue types included in the GSE9954 data series. Each row represents one gene, with a total of 202 adipose-specific genes. Each column represents one tissue sample (GEO sample number), including 3 epididymal adipose, 3 liver, 3 diaphragm, 3 salivary gland, 3 spleen, 4 muscle, 3 brain, 3 lung, 3 kidney, 3 adrenal gland, 4 bone marrow, 5 pituitary gland, 3 seminal vesicle, 3 thymus, 3 testis, 3 heart, 3 small intestine and 3 eye tissue samples. Relative gene expression is indicated according to the color scale, where red indicates elevated expression and blue indicates reduced expression. Gene expression values were transformed into $\log _{2}$-based values (distribution range, 6 and 14 ).

analyses were performed using the online software DAVID (https://david.ncifcrf.gov/) (19) and KOBAS (http://kobas.cbi. pku.edu.cn/) (20,21). The Gene Ontology (GO) and Kyoto Encyclopedia of Genes and Genomes (KEGG) pathway analyses were used as ontology sources. Significant enrichment was considered at $\mathrm{P}<0.01$.

PPI network and module analysis. The assessment of PPI information and the construction of a functional protein association network were performed using the Search Tool for the Retrieval of Interacting Genes/Proteins (STRING) database (version 10.5; $\mathrm{http}: / / \mathrm{string}-\mathrm{db} .0 \mathrm{~g} /$ /) (22). To evaluate the intensity of interaction among the input genes, the genes were mapped in STRING using a combined score (experimentally validated interactions) of $>0.4$ as the cut-off criterion. Next, the PPI network was constructed and visualized with Cytoscape software (version 3.6.0; http://www.cytoscape.org/) (23). The MCODE plug-in (http://apps.cytoscape.org/apps/mcode) (24) was used to scan the PPI network in order to identify densely connected regions.

Gene co-expression network analysis. Weighted gene co-expression network analysis (WGCNA) can be used to describe the network of gene expression estimates based on a correlation (25). Highly connected nodes in the co-expression network are often considered as key components affecting the expression of the whole network. In the current study, co-expression network analysis was performed using the WGCNA package (https://cran.r-project. org/web/packages/WGCNA/index.html) (25), with a threshold for weight set at $>0.6$.

\section{Results}

Identification of adipose-specific genes. First, the raw data of GSE9954 in text format were downloaded from the GEO database. Gene expression values were normalized and imputed using the packages impute and limma in R. The gene expression profiles of adipose tissue were compared with those of 17 other types of non-adipose tissues in order to identify DEGs, employing an adjusted P-value of $<0.05$ and a $\log \mathrm{FCl}$ of $>0.5$ as the cut-off criteria. The overlap of these comparative results revealed 219 DEGs which were commonly upregulated (215) or downregulated (4) in adipose tissue compared with all the other tissues (Table I). Next, 13 genes that could not be recognized were excluded, and the remaining 202 common upregulated genes were considered to be adipose-specific genes. These included several important genes, such as acetyl-CoA carboxylase $\alpha$ (Acaca), peroxisome proliferator-activated receptor $\gamma$ (Pparg) and leptin (Lep), as well as adiponectin, C1Q and collagen domain containing (Adipoq), and fatty acid synthase (Fasn). Employing the heatmap package in the $\mathrm{R}$ programming language, a heat map of the adipose-specific genes was developed using gene expression data from the 58 samples of 18 tissue types included in the data series (Fig. 1).

Process and pathway enrichment analyses. To investigate the role of the adipose-specific genes in WAT, process and pathway enrichment analyses were performed. The top 20 significant GO biological processes were selected by DAVID online software (Fig. 2A). According to the results, adipogenesis-associated biological processes, including brown 
A

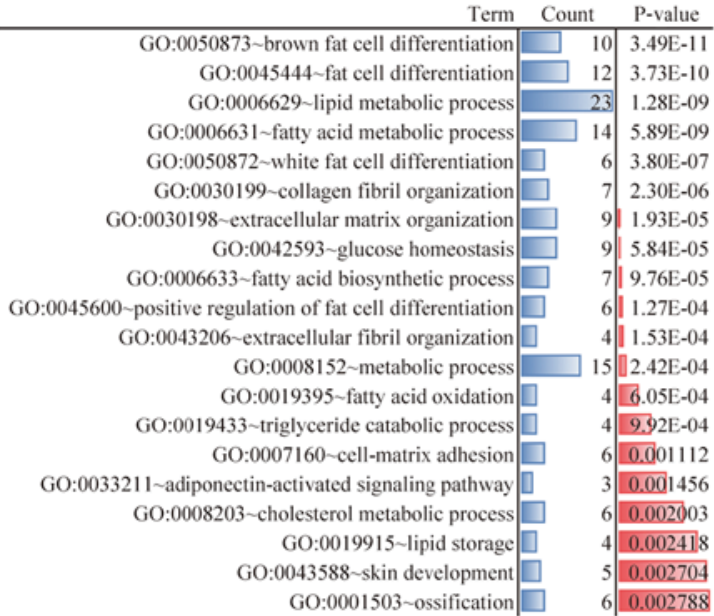

C

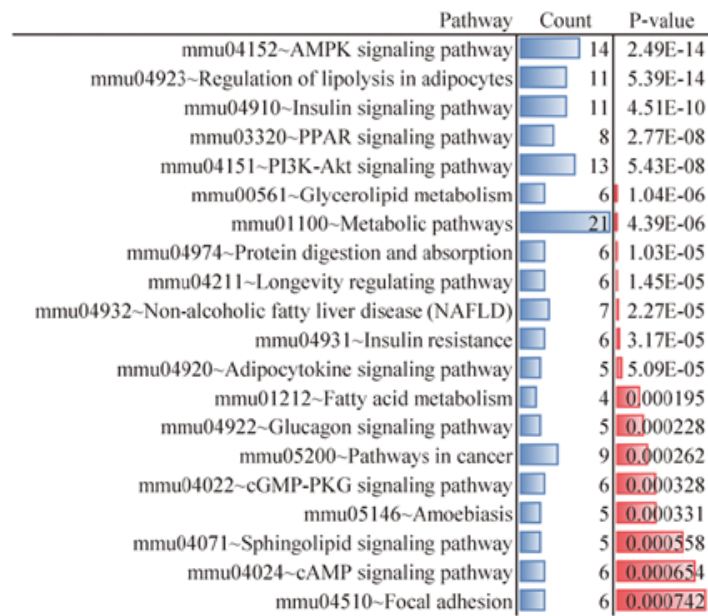

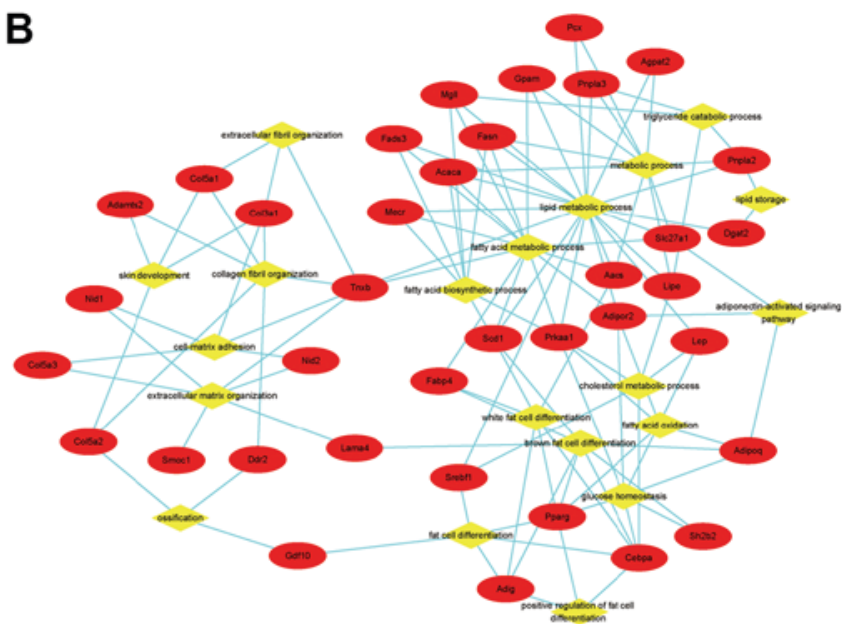

D

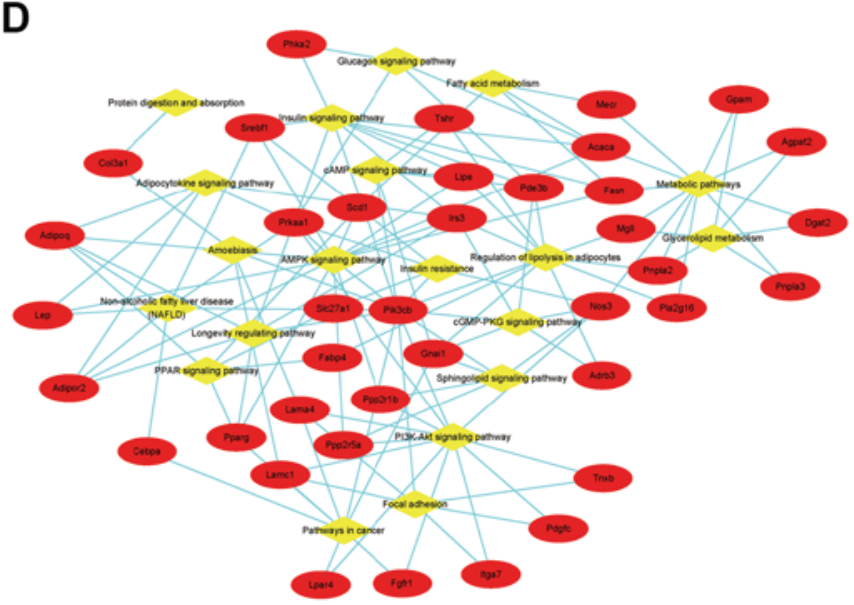

Figure 2. GO biological processes and KEGG pathways analyses of adipose-specific genes. (A) GO annotations of the adipose-specific genes with the top 20 enrichment scores for biological processes. (B) Interactions and overlap of associated molecules among the top 20 biological processes. (C) KEGG pathway enrichment analysis of the adipose-specific genes with the top 20 enrichment scores. (D) Interaction and overlap of associated molecules among the top 20 pathways. Ellipses represent the most significantly enriched biological processes or pathways, while diamonds indicate the genes that act as a link hinge between biological processes or pathways. GO, Gene Ontology; KEGG, Kyoto Encyclopedia of Genes and Genomes.

fat cell differentiation, fat cell differentiation and white fat cell differentiation respectively displayed the highest, second highest and fourth highest significance, while lipid metabolic processes were considered the central biological process category according to the exchanges among the terms (Fig. 2B). Therefore, fat cell differentiation and lipid metabolic processes are considered to be key biological processes in WAT. Furthermore, the top 20 significant KEGG pathways were identified by KOBAS online software (Fig. 2C). The highest statistical significance was displayed by the adenine monophosphate-activated protein kinase (AMPK) signaling pathway, which was simultaneously considered as the central pathway according to the exchanges among the pathways (Fig. 2D). Therefore, the AMPK signaling pathway is considered to be a key pathway in WAT.

PPInetworkanalysisandmodule screening.The adipose-specific genes were uploaded to the STRING database, and a total of 118 nodes and 336 edges were obtained with a combined score of $>0.4$ (Fig. 3A). Acaca was the hub gene in the PPI network, exhibiting the highest node degree (Fig. 3B). Further analysis of the top 30 genes using the MCODE plug-in revealed two significant modules: Module 1 (consisting of 17 genes) and module 2 (consisting of 8 genes) (Fig. 3C). Through KEGG pathway analysis, module 1 was found to be significantly enriched for the AMPK, PPAR and insulin signaling pathways, and module 2 was found to be significantly enriched in protein digestion and absorption. Notably, the top 13 genes (76\% of module 1 genes) exhibiting the highest degrees in the PPI network were all classified into module 1 . This analysis demonstrated that module 1 was the core cluster in WAT, and that the key genes in WAT included 17 genes from module 1. These genes were Acaca, Pparg, Lep, Adipoq, Fasn, lipase (Lipe), sterol regulatory element-binding transcription factor 1 (Srebf1), diacylglycerol O-acyltransferase 2 (Dgat2), patatin-like phospholipase domain containing 2 (Pnpla2), complement factor D (Cfd), resistin (Retn), fatty acid-binding protein 4 (Fabp4), cell death-inducing DFFA-like effector c (Cidec), CCAAT/enhancer-binding protein $\alpha$ (Cebpa), solute carrier family 27 member 1 (Slc27a1), glycerol-3-phosphate acyltransferase mitochondrial (Gpam), and ATP-binding cassette sub-family A member 1 (Abcal).

Co-expression network analysis. In the aforementioned analysis, the key genes in WAT were screened based on STRING database. However, in order to determine whether 

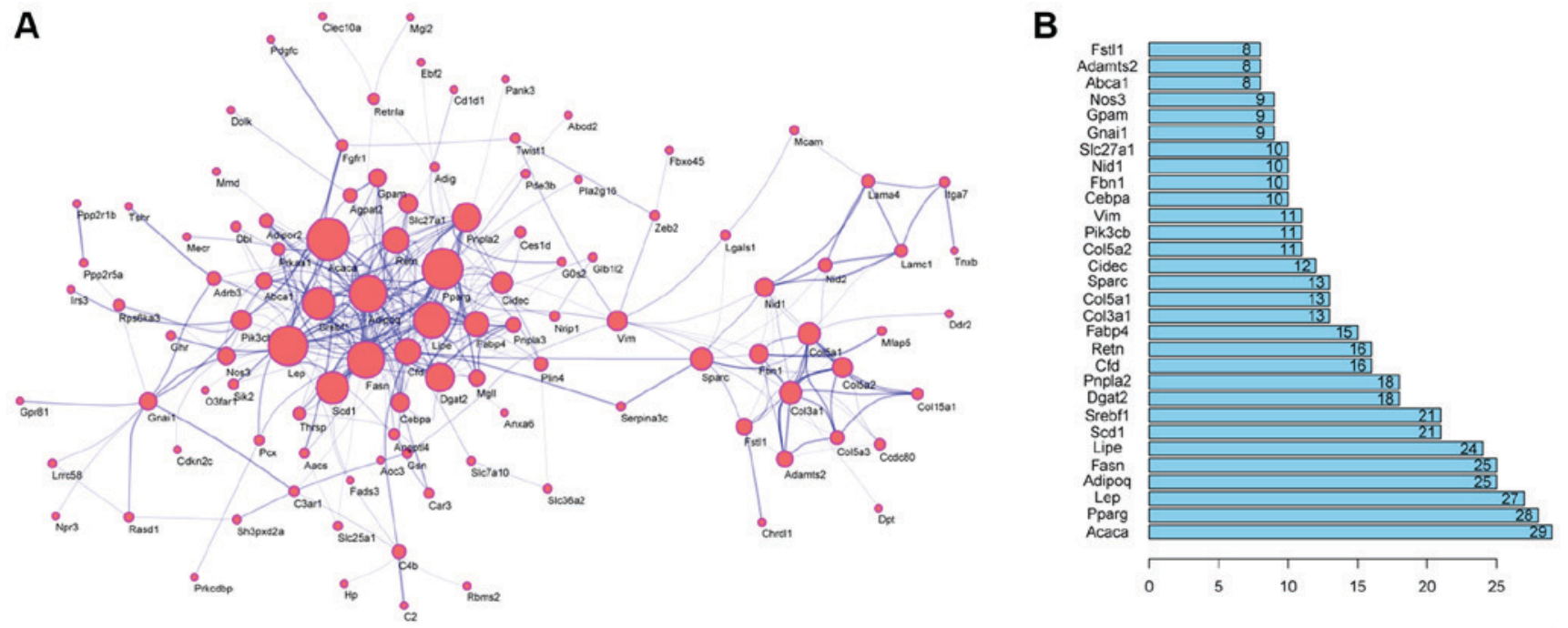

C

Module 1

Module 2

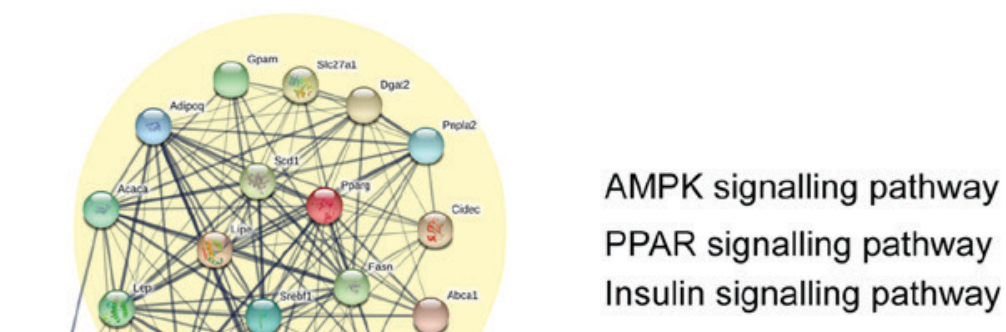

Protein digestion and absorption

Figure 3. PPI network complex and MCODE module analysis of adipose-specific genes. (A) The PPI network was visualized using Cytoscape software (isolated nodes were not included). Node size represents the node degree (a larger size indicates a higher degree). The width and transparency of the edge represent the combined score of the edge (a wider or more opaque edge indicates a higher combined score). (B) Top 30 genes with the highest node degrees. (C) MCODE module screening for the top 30 genes and pathway enrichment analysis for the modules. PPI, protein-protein interaction; MCODE, molecular complex detection; AMPK, adenine monophosphate-activated protein kinase; PPAR, peroxisome proliferator-activated receptor.

other key candidate genes were excluded from these results, co-expression analysis of the adipose-specific genes in the 58 tissue samples from 18 types of mouse tissues was further performed using the WGCNA package of the R programming language. Next, a co-expression network was constructed and visualized using Cytoscape software, according to the weight between the genes (Fig. 4A). Amine oxidase copper containing 3 (Aoc3) and adrenoceptor beta 3 (Adrb3) were the top 2 highly connected nodes in the network according to the number of co-expressed genes. Furthermore, Aoc3 was strongly positively correlated with the key genes Lep (Pearson's correlation coefficient: $r=0.956)$, Retn $(r=0.935)$ and Cidec $(r=0.92)$, while a strong positive correlation of Adrb3 with Lep $(r=0.958)$, Retn $(r=0.937)$ and Cidec $(r=0.906)$ was also observed (Fig. 4B). These results suggested that Aoc3 and Adrb3 may serve an important role in WAT development, and thus these two genes may be potential key candidate genes in WAT.

\section{Discussion}

In the present study, to accurately investigate the underlying molecular mechanisms of WAT, 202 adipose-specific genes were identified through comparative transcriptome analysis of multiple tissues. The expression level of these genes was found to be higher in adipose tissue as compared with that in the 17 other tissue types examined. This gene set contains numerous well-known adipose marker genes, such as Acaca, Pparg, Lep, Adipoq and Fasn. In addition, several other genes were identified, of which the role in fat has not been widely reported, such as Adamts2, Fstl1, Fbn1 and Sparc. Subsequent analysis was based on the 202 adipose-specific genes. 
A

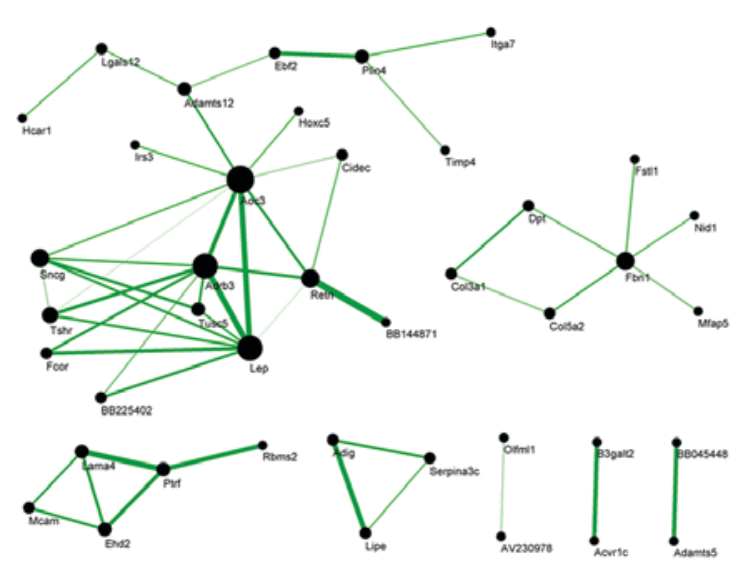

B
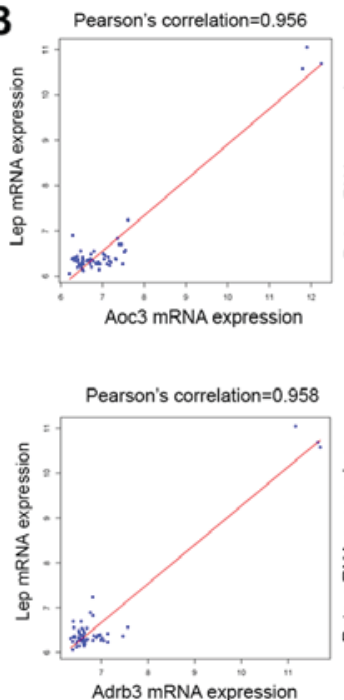
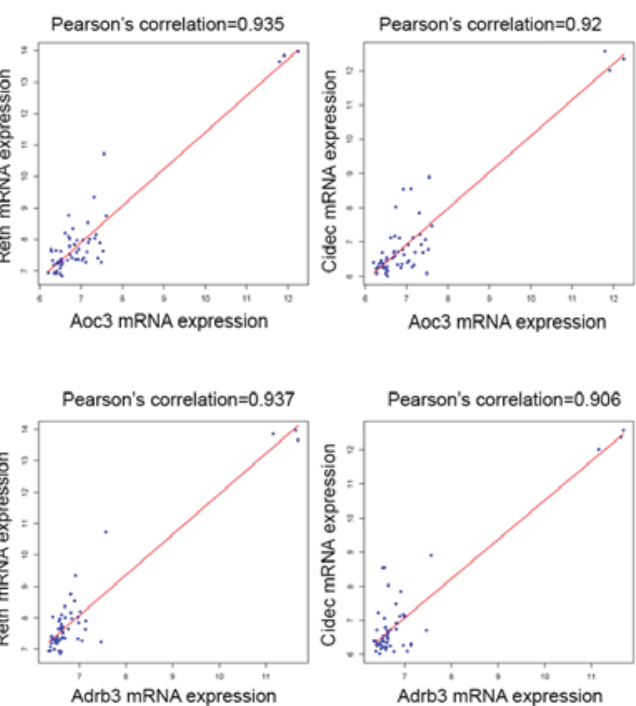

Figure 4. Construction of a co-expression network and identification of key candidate genes. (A) Co-expression network of adipose-specific genes. Node size represents the node degree (a larger size indicates a higher degree). The width and transparency of the edge represent the weight score of the edge (a wider or more opaque edge indicates a higher weight score). (B) Aoc3 and Adrb3 were strongly positively correlated with the key genes Lep, Retn and Cidec in 58 tissue samples from 18 types of mouse tissues. Aoc3, amine oxidase copper containing 3; Adrb3, adrenoceptor beta 3; Lep, leptin; Retn, resistin; Cidec, cell death-inducing DFFA-like effector c.

Process and pathway enrichment analyses were also performed for the adipose-specific genes in the current study. In WAT, the key biological processes are fat cell differentiation and lipid metabolic process, while the AMPK signaling pathway is the key pathway involved. During WAT development, fat cell differentiation confers adipocytes and adipose tissue with the typical functional properties associated with systemic metabolism (26). Preadipocytes confer adipose tissue with permanent functional plasticity for adipose expansion through transformation into differentiated adipocytes (26). A series of transcription factors cooperate with cell cycle proteins, manage adipogenic gene expression and give rise to adipocyte development during adipogenesis (27). In addition, triacylglycerol synthesis and degradation-associated enzymes acquire increasing activity and expression levels, triggered by a cascade of transcription factors in the terminal phase of differentiation (11). Glucose and fatty acid transporters and insulin receptors also demonstrate increased levels (11). Beyond the classical energy storage form of adipocytes, the synthesis of adipocyte-secreted hormones and cytokines confers adipocytes as extremely distinctive endocrine cells, which serve an important role in various physiological activities $(9,26)$. One distinct characteristic of adipogenesis is the evident change in morphology from a fibroblastic to spherical shape. In mature adipocytes, single large lipid droplets ultimately occupy most of the cell volume, and the nuclei and cytoplasm are pushed to the periphery. Thus, fat cell differentiation confers lipid metabolic abilities, including energy storage and mobilization abilities, upon WAT (22). As an important regulator of energy metabolism in WAT, AMPK activation inhibits fatty acid synthesis by downregulating key lipogenic enzymes (28), while promoting free fatty acid oxidation (29).

In order to explore key genes in WAT, the construction of a PPI network of adipose-specific genes and further analyses were conducted in the present study. It was observed that Acaca, Pparg and Lep were hub nodes in the PPI network, and that there were two modules in the PPI network. Module 1 exhibited more highly interconnected nodes with higher significance, and was predicted to be the core cluster maintaining WAT development and performance. In addition, module 1 mainly involved the AMPK, PPAR and insulin signaling pathways. The 17 genes included in module 1 are known to participate in the development and various functions of WAT, including the transcriptional regulation of adipogenesis (Pparg, Cebpa and Srebf1) (30), endocrine functions of adipose tissue (Lep, Adipoq, Cfd, Retn and Abca1) (31-33), de novo fatty acid synthesis (Fasn and Acaca) (34), fatty acid transport (Fabp4 and Slc27a1) $(35,36)$, triacylglycerol synthesis (Gpam, Dgat2 and Cidec) (37-39), and lipolysis and its regulation (Lipe and Pnpla2) (40). Based on the aforementioned results, the 17 key genes identified stand out and are reliable, as demonstrated by further screening of the node degrees and MCODE modules of the PPI network.

The STRING database contains known and predicted interactions between proteins based on experiments, text mining, database contents and bioinformatics methods. Therefore, the current study next investigated whether there were any other key genes in addition to these 17 key genes that were overlooked due to their lack of priority in the STRING PPI network. To identify other key candidate genes, a co-expression analysis of adipose-specific genes was performed using the expression data from 58 samples from 18 mouse tissues. The results revealed that Aoc3 and Adrb3 were communication hubs in the co-expression network. In particular, Aoc3 and Adrb3 were strongly positively correlated with the key genes Lep, Retn and Cidec. Thus, artificially altering the expression levels of Aoc3 and Adrb3, as highly connected hubs, may influence the expression patterns of numerous other adipose-specific genes or communicate changes that occur elsewhere in the co-expression network, leading to the dysfunction of adipose tissue. The Aoc 3 gene encodes the enzyme vascular adhesion protein (VAP-1), which is a 
member of the semicarbazide-sensitive amine oxidase family and primarily localizes to the cell surface on the adipocyte plasma membrane (41). VAP-1 is associated with a number of vascular diseases (42), and may be involved in adipogenesis and obesity (43). Furthermore, the Adrb3 gene encodes a beta-adrenergic receptor that responds to noradrenaline and mediates lipolysis in adipocytes (44). Trp64Arg (rs 4994), a single nucleotide polymorphism of Adrb3, is associated with glucose homeostasis, insulin sensitivity and obesity (45). Based on the aforementioned information, it can be suggested that Aoc3 and Adrb3 may be key candidate genes for the function of WAT.

In conclusion, 202 adipose specific genes were identified in the current study by systematic comparative analysis of multiple tissues at the transcriptome level. Several well-known genes, as well as other genes with few studies reporting their role in fat, were identified. Thus, the present study provided an important target list for future WAT development studies. Subsequently, the function of these genes as a whole was examined by functional enrichment analysis, and construction of PPI and co-expression networks. Key processes, pathways and genes that may serve vital roles in the development and physiological function of WAT were identified. Taken together, the current study indicated several helpful targets for future research into the molecular mechanisms and potential diagnostic biomarkers of obesity-associated diseases caused by adipose dysfunction. However, further experiments at the molecular and cellular levels are required to verify the function of the identified genes.

\section{Acknowledgements}

Not applicable.

\section{Funding}

The present study was supported by grants from the National Science and Technology Support Projects (no. 2015BAD03B04), the National Beef Yak Industry Technology System (no. CARS-37), and the Major Agricultural Science and Technology Innovation and Transformation Plan in Shaanxi Province (no. NYKJ-2016-06).

\section{Availability of data and materials}

All data generated or analyzed during this study are included in the published article.

\section{Author's contributions}

LZ, SZ and LW conceived and designed the study. SZ performed data analysis and wrote the manuscript. The data analysis in this study was largely provided by LW, who also provided constructive suggestions for the manuscript and language modification.

\section{Ethics approval and consent to participate}

Not applicable.

\section{Patient consent for publication}

Not applicable.

\section{Competing interests}

The authors declare that they have no competing interests.

\section{References}

1. World Health Organization. Obesity and overweight. http://www. who.int/news-room/fact-sheets/detail/obesity-and-overweight. Accessed March 8, 2018.

2. Hajer GR, van Haeften TW and Visseren FL: Adipose tissue dysfunction in obesity, diabetes, and vascular diseases. Eur Heart J 29: 2959-2971, 2008.

3. Oda E: Historical perspectives of the metabolic syndrome. Clin Dermatol 36: 3-8, 2018.

4. Moreno-Navarrete JM and Fernández-Real JM: Adipocyte differentiation. In: Adipose tissue biology. 'Vol' Springer pp 69-90, 2017

5. Ali AT, Hochfeld WE, Myburgh R and Pepper MS: Adipocyte and adipogenesis. Eur J Cell Biol 92: 229-236, 2013.

6. Spalding KL, Arner E, Westermark PO, Bernard S, Buchholz BA, Bergmann O, Blomqvist L, Hoffstedt J, Näslund E, et al: Dynamics of fat cell turnover in humans. Nature 453: 783-787, 2008.

7. Gustafson B, Hedjazifar S, Gogg S, Hammarstedt A and Smith U: Insulin resistance and impaired adipogenesis. Trends Endocrin Metab 26: 193-200, 2015.

8. Hirsch J and Batchelor B: Adipose tissue cellularity in human obesity. Clin Endocrinol Metab 5: 299-311, 1976.

9. Ahima RS and Flier JS: Adipose tissue as an endocrine organ. Trends Endocrinol Metab 11: 327-332, 2000.

10. Ducharme NA and Bickel PE: Lipid droplets in lipogenesis and lipolysis. Endocrinology 149: 942-949, 2008.

11. Gregoire FM, Smas CM and Sul HS: Understanding adipocyte differentiation. Physiol Rev 78: 783-809, 1998.

12. Barrett T, Suzek TO, Troup DB, Wilhite SE, Ngau WC, Ledoux P, Rudnev D, Lash AE, Fujibuchi W and Edgar R: NCBI GEO: Mining millions of expression profiles-database and tools. Nucleic Acids Res 33 (Database Issue): D562-D566, 2005.

13. Kaplan S, Boztepe S and Arikoglu H: The potential of microarray databases to identify tissue specific genes. Kafkas Univ Vet Fak 22: 29-35, 2016.

14. Ullah M, Stich S, Häupl T, Eucker J, Sittinger M and Ringe J: Reverse differentiation as a gene filtering tool in genome expression profiling of adipogenesis for fat marker gene selection and their analysis. PLoS One 8: e69754, 2013.

15. Fujioka S, Matsuzawa Y, Tokunaga K, Kawamoto T, Kobatake T, Keno Y, Kotani K, Yoshida S and Tarui S: Improvement of glucose and lipid metabolism associated with selective reduction of intra-abdominal visceral fat in premenopausal women with visceral fat obesity. Int J Obes 15: 853-859, 1991.

16. Thorrez L, Van Deun K, Tranchevent LC, Van Lommel L, Engelen K, Marchal K, Moreau Y, Van Mechelen I and Schuit F: Using ribosomal protein genes as reference: A tale of caution. PLoS One 3: e1854, 2008.

17. Hastie T, Tibshirani R, Narasimhan B and Chu G: Impute: Imputation for microarray data. Bioinformatics 17: 520-525, 2001.

18. Ritchie ME, Phipson B, Wu D, Hu Y, Law CW, Shi W and Smyth GK: limma powers differential expression analyses for RNA-sequencing and microarray studies. Nucleic Acids Res 43: e47, 2015

19. Tripathi S, Pohl MO, Zhou Y, Rodriguez-Frandsen A, Wang G, Stein DA, Moulton HM, DeJesus P, Che J, Mulder LC, et al: Meta- and orthogonal integration of influenza 'OMICs' data defines a role for UBR4 in virus budding. Cell Host Microbe 18: 723-735, 2015.

20. Wu J, Mao X, Cai T, Luo J and Wei L: KOBAS server: A web-based platform for automated annotation and pathway identification. Nucleic Acids Res 34: W720-W724, 2006.

21. Xie C, Mao X, Huang J, Ding Y, Wu J, Dong S, Kong L, Gao G, Li CY and Wei L: KOBAS 2.0: A web server for annotation and identification of enriched pathways and diseases. Nucleic Acids Res 39: W316-W322, 2011. 
22. Franceschini A, Szklarczyk D, Frankild S, Kuhn M, Simonovic M Roth A, Lin J, Minguez P, Bork P, von Mering C and Jensen LJ: STRING v9.1: Protein-protein interaction networks, with increased coverage and integration. Nucleic Acids Res 41 (Database Issue): D808-D815, 2013.

23. Shannon P, Markiel A, Ozier O, Baliga NS, Wang JT, Ramage D, Amin N, Schwikowski B and Ideker T: Cytoscape: A software environment for integrated models of biomolecular interaction networks. Genome Res 13: 2498-2504, 2003.

24. Bader GD and Hogue CW: An automated method for finding molecular complexes in large protein interaction networks. BMC Bioinformatics 4: 2, 2003.

25. Langfelder $P$ and Horvath S: WGCNA: An R package for weighted correlation network analysis. BMC Bioinformatics 9: $559,2008$.

26. Vázquez-Vela ME, Torres $\mathrm{N}$ and Tovar AR: White adipose tissue as endocrine organ and its role in obesity. Arch Med Res 39: 715-728, 2008.

27. Rosen ED, Walkey CJ, Puigserver P and Spiegelman BM: Transcriptional regulation of adipogenesis. Genes Dev 14: 1293-1307, 2000.

28. Fernández-Galilea M, Pérez-Matute P, Prieto-Hontoria PL, Sáinz N, López-Yoldi M, Houssier M, Martínez JA, Langin D and Moreno-Aliaga MJ: $\alpha$-lipoic acid reduces fatty acid esterification and lipogenesis in adipocytes from overweight/obese subjects. Obesity (Silver Spring) 22: 2210-2215, 2014.

29. Minokoshi Y, Kim YB, Peroni OD, Fryer LG, Müller C, Carling D and Kahn BB: Leptin stimulates fatty-acid oxidation by activating AMP-activated protein kinase. Nature 415 : 339-343, 2002.

30. Rosen ED and MacDougald OA: Adipocyte differentiation from the inside out. Nat Rev Mol Cell Biol 7: 885-896, 2006.

31. Song NJ, Kim S, Jang BH, Chang SH, Yun UJ, Park KM, Waki H, Li DY, Tontonoz P and Park KW: Small molecule-induced complement factor D (Adipsin) promotes lipid accumulation and adipocyte differentiation. PLoS One 11: e162228, 2016.

32. Meier U and Gressner AM: Endocrine regulation of energy metabolism: Review of pathobiochemical and clinical chemical aspects of leptin, ghrelin, adiponectin, and resistin. Clin Chem 50: 1511-1525, 2004

33. Damen MSMA, Dos Santos JC, Hermsen R, Adam van der Vliet J, Netea MG, Riksen NP, Dinarello CA, Joosten LAB and Heinhuis B: Interleukin-32 upregulates the expression of ABCA1 and ABCG1 resulting in reduced intracellular lipid concentrations in primary human hepatocytes. Atherosclerosis 271: 193-202, 2018

34. Tanos R, Murray IA, Smith PB, Patterson A and Perdew GH: Role of the Ah receptor in homeostatic control of fatty acid synthesis in the liver. Toxicol Sci 129: 372-379, 2012.
35. Stahl A: A current review of fatty acid transport proteins (SLC27). Pflugers Arch 447: 722-727, 2004.

36. Hertzel AV and Bernlohr DA: The mammalian fatty acid-binding protein multigene family: Molecular and genetic insights into function. Trends Endocrinol Metab 11: 175-180, 2000.

37. Smith SJ, Cases S, Jensen DR, Chen HC, Sande E, Tow B, Sanan DA, Raber J, Eckel RH and Farese RV Jr: Obesity resistance and multiple mechanisms of triglyceride synthesis in mice lacking Dgat. Nat Genet 25: 87-90, 2000.

38. Takeuchi K and Reue K: Biochemistry, physiology, and genetics of GPAT, AGPAT, and lipin enzymes in triglyceride synthesis. Am J Physiol Endocrinol Metab 296: E1195-E1209, 2009.

39. Li Y, Kang H, Chu Y, Jin Y, Zhang L, Yang R, Zhang Z, Zhao S and Zhou L: Cidec differentially regulates lipid deposition and secretion through two tissue-specific isoforms. Gene 641: 265-271, 2018.

40. Reilich P, Horvath R, Krause S, Schramm N, Turnbull DM, Trenell M, Hollingsworth KG, Gorman GS, Hans VH, Reimann J, et al: The phenotypic spectrum of neutral lipid storage myopathy due to mutations in the PNPLA2 gene. J Neurol 258 1987-1997, 2011

41. Hernandez-Guillamon M, Solé M, Delgado P, García-Bonilla L, Giralt D, Boada C, Penalba A, García S, Flores A, Ribó M, et al: VAP-1/SSAO plasma activity and brain expression in human hemorrhagic stroke. Cerebrovasc Dis 33: 55-63, 2012.

42. Schilter HC, Collison A, Russo RC, Foot JS, Yow TT, Vieira AT, Tavares LD, Mattes J, Teixeira MM and Jarolimek W: Effects of an anti-inflammatory VAP-1/SSAO inhibitor, PXS-4728A, on pulmonary neutrophil migration. Resp Res 16: 42, 2015.

43. Bour S, Daviaud D, Gres S, Lefort C, Prévot D, Zorzano A, Wabitsch M, Saulnier-Blache J, Valet $\mathrm{P}$ and Carpéné C: Adipogenesis-related increase of semicarbazide-sensitive amine oxidase and monoamine oxidase in human adipocytes. Biochimie 89: 916-925, 2007

44. Manolio TA, Collins FS, Cox NJ, Goldstein DB, Hindorff LA, Hunter DJ, McCarthy MI, Ramos EM, Cardon LR, Chakravarti A, et al: Finding the missing heritability of complex diseases. Nature 461: 747-753, 2009.

45. Nakashima H, Omae K, Nomiyama T, Yamano Y, Takebayashi T and Sakurai Y: Beta-3-adrenergic receptor Trp64Arg polymorphism: Does it modulate the relationship between exercise and percentage of body fat in young adult Japanese males? Environ Health Prev Med 18: 323-329, 2013. International (CC BY-NC-ND 4.0) License. 